\title{
Structure and Function of Nano-sized InSb Precipitate Embedded in an Al Alloy
}

\author{
Yong Zhang ${ }^{1}$, Xiang Gao ${ }^{1}$, Nikhil V. Medhekar ${ }^{1}$ and Laure Bourgeois ${ }^{1,2}$ \\ 1. Department of Materials Science and Engineering, Monash University, Melbourne, Australia \\ 2. Monash Centre for Electron Microscopy, Monash University, Melbourne, Australia
}

$\mathrm{Al}-\mathrm{Cu}$ alloys are the most typical ageing hardenable aluminium alloys and have achieved wide applications ranging from the aircraft to the automobile industries, which trace back to 100 years ago [1]. Microalloying solutes (usually $<0.1$ at.\%) including $\mathrm{Sn}$, In and $\mathrm{Cd}$ are hypothesized to form nanosized precipitates first, and then catalyse the heterogeneous nucleation of $\theta^{\prime}\left(\mathrm{Al}_{2} \mathrm{Cu}\right)$, a nanoscale precipitate and the dominant strengthening precipitate in $\mathrm{Al}-\mathrm{Cu}$ alloys [2]. However, to date, there are no solid experimental results showing how $\theta^{\prime}$ nucleates heterogeneously from other precipitates at the atomic level because it is challenging to capture a nucleation process [3, 4]. In this study, using diffraction, imaging and X-ray energy dispersive spectroscopy in scanning transmission electron microscopy (STEM), we show that nano-sized InSb particles adopting a new crystal structure catalyse the heterogonous nucleation of $\theta^{\prime}$ by surface absorption. The nanoscale InSb precipitates are found to adopt a face-centred cubic (FCC) crystal structure that different from the Zinc Blende structure of the bulk state.

Fig. 1 (a) shows the formation of a truncated octahedral particle which is decorated by single layer $\mathrm{Cu}$ atoms (Guinier-Preston zone, GP zone) at some surfaces. The single layer $\mathrm{Cu}$ grows into double layers $\left(\theta^{\prime \prime}\right.$, in (b)) and a plate $\left(\theta^{\prime}\right.$, in $\left.(\mathrm{c})\right)$, which unambiguously shows how $\theta^{\prime}$ nucleates heterogeneously from the pre-existing nanoscale particles step by step. The octahedral nanoparticles are composed of In and $\mathrm{Sb}$ elements (Fig. 2). However, the two dimensional moiré fringes shown in Fig. 1 are inconsistent with any known InSb phases. Nanodiffraction in STEM mode was used to determine the crystal structure of the InSb nanoparticle. The diffraction patterns shown in Fig. 3 (a) suggest the nanoparticle adopts a FCC structure. As shown in all these images, we have been unable to detect any chemical ordering in the nanoscale precipitates. The determined crystal structure is consistent with the moire fringes and selected area diffraction patterns from a bigger particle (Fig. 3 (b)). The bigger particle was found after1 month ageing, which is far from the nucleation stage of $\theta^{\prime}$. Density functional theory calculations give nearly the same lattice constant, $\mathrm{a}=0.48 \mathrm{~nm}$. Simulated diffraction patterns based on chemically disordered structures are consistent with the experimental observations (Fig. 3 (c)).

References:

[1] F.W. Gayle, et al, Science 266 (1994) p. 1015-1017.

[2] L. Bourgeois, et al, Acta Mater. 60 (2012) p. 633-644.

[3] U. Kaiser, et al, Nature Materials 1 (2002) p. 102-105.

[4] S.K. Malladi, et al, Nano Lett. 14 (2013) p. 384-389.

[5] The authors acknowledge funding from the Australian Research Council (DP150100558 and LE0454166), computational support from the Monash Sun Grid cluster, the National Computing Infrastructure and Pawsey Supercomputing Centre funded by the Australian Government, and the use of facilities within the Monash Centre for Electron Microscopy. 

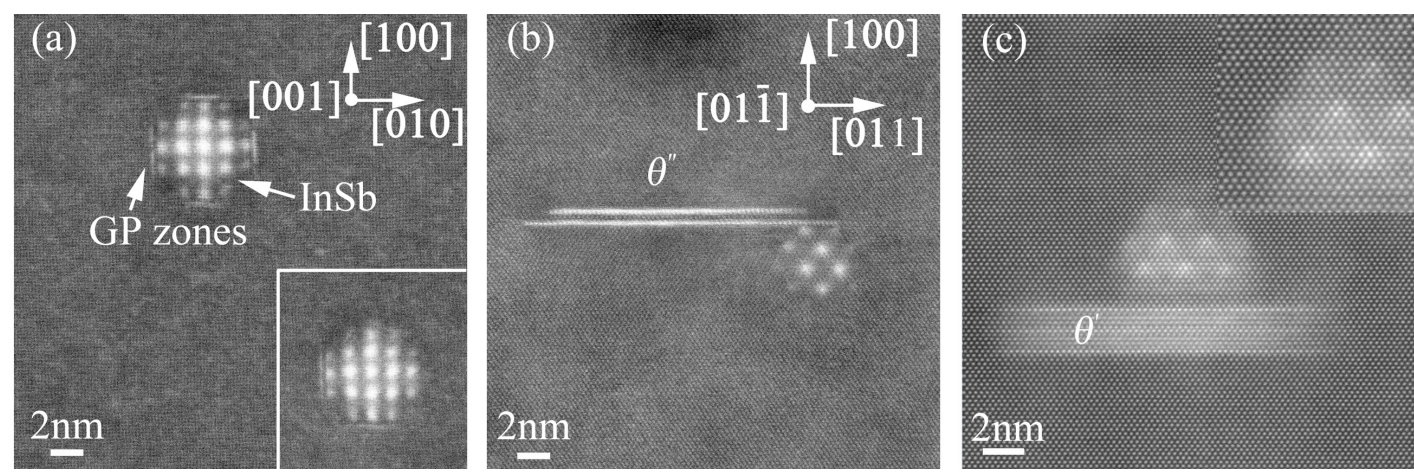

Figure 1. Cu-rich phases nucleate at the interface of $\{002\}_{\mathrm{Al}} /\{001\}_{\mathrm{InSb}}$ in $\mathrm{Al}-1.7 \mathrm{Cu}-0.025 \mathrm{In}-0.025 \mathrm{Sb}$ (at. \%) alloy aged at $200{ }^{\circ} \mathrm{C}$ for $10 \mathrm{~min}$.
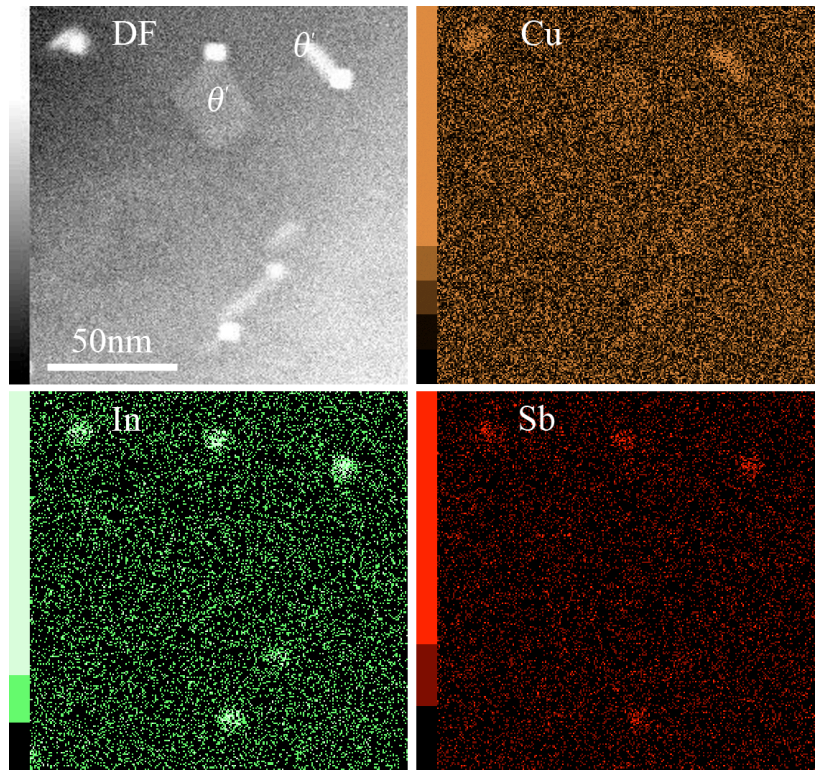

Fig. 2. X-ray energy dispersive spectroscopy mapping showing the $\mathrm{Cu}$-rich phases attached to $\mathrm{InSb}$ crystals in the alloy aged for $30 \mathrm{~min}$ at $200{ }^{\circ} \mathrm{C}$.
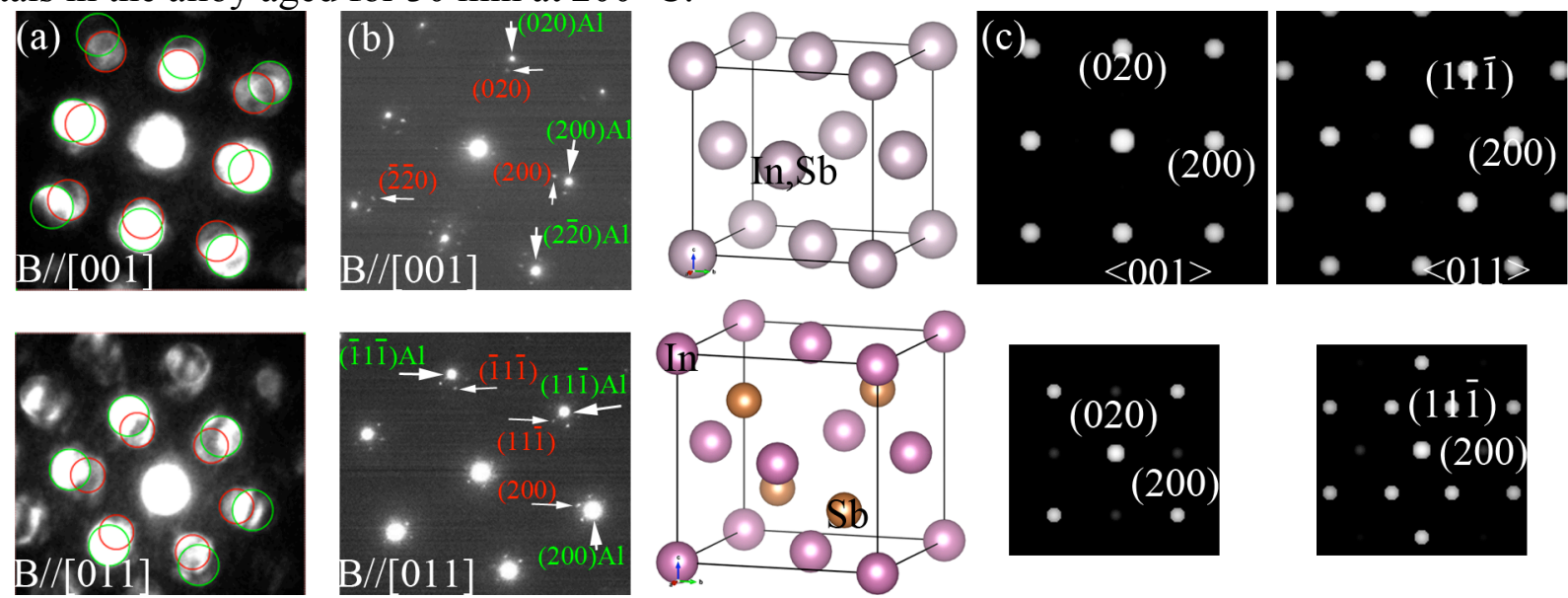

Figure 3. (a) nanodiffraction patterns with the beam focusing on a $\sim 8 \mathrm{~nm}$ InSb particle formed after $2 \mathrm{~h}$ ageing at $200{ }^{\circ} \mathrm{C}$ (B in insets meaning incident beam), (b) SAED patterns with the aperture on a $\sim 50 \mathrm{~nm}$ InSb particle formed after 30 days ageing at $300{ }^{\circ} \mathrm{C}$ (right), (c) crystal structure of FCC InSb (up) and Zinc Blende InSb (bottom) and the simulated diffraction patterns. 